\title{
Stop and go: Oocyten auf ihrem Weg zu einer befruchtungsfähigen Eizelle
}

ANDREAS HEIM, THOMAS U. MAYER

KONSTANZ RESEARCH SCHOOL CHEMICAL BIOLOGY, UNIVERSITÄT KONSTANZ

Fertilization, the fusion of the female and male gamete, is the initiating event for the development of any sexually-reproducing organism. The production of haploid gametes involves two consecutive meiotic divisions during which the diploid genome is reduced to half, creating four haploid cells. Here, we discuss how the interplay of dynamic protein phosphorylation and irreversible protein degradation drives meiotic cell cycle regulation in vertebrate oocytes.

DOI: $10.1007 / \mathrm{s} 12268-020-1330-5$

(c) Die Autoren 2020

Durch das Verschmelzen der haploiden männlichen und weiblichen Keimzellen (Gameten) kommt es zur Ausbildung der Zygote, die über einen vollständigen diploiden Chromosomensatz verfügt. Bei einer menschlichen Zygote entspricht dies 46 Chromosomen, bestehend aus 23 mütterlichen und 23 väterlichen Chromosomen. Im Anschluss an die Fertilisation führt ein komplexes Programm aus mitotischen Teilungen, Differenzierungs- und Entwicklungsprozessen zu der Entwicklung eines Embryos. Schon während der frühen Embryonalentwicklung kommt es in Säugetieren in Abwesenheit eines Y-Chromosoms zur Ausbildung weiblicher Gameten, den Oocyten.

\section{Entwicklung weiblicher Gameten}

Während der frühen Entwicklungsphase weiblicher Embryos wandern Urkeimzellen in die sich bildenden Eierstöcke, differenzieren sich zu Ureizellen (Oogonien) und durchlaufen eine Vielzahl mitotischer Teilungen. Am Ende dieser Vermehrungsphase liegen beim Menschen ca. sieben Millionen Oogonien vor, von denen die große Mehrzahl noch während der Embryonalentwicklung abstirbt. Tatsächlich liegen bei der Geburt nur noch etwa ein bis zwei Millionen Oogonien vor, von denen nur ca. 400.000 bis zur Pubertät überleben und von denen wiederum nur 300 bis 500 sich zu befruchtungsfähigen Eizellen entwickeln [1]. Während sich die

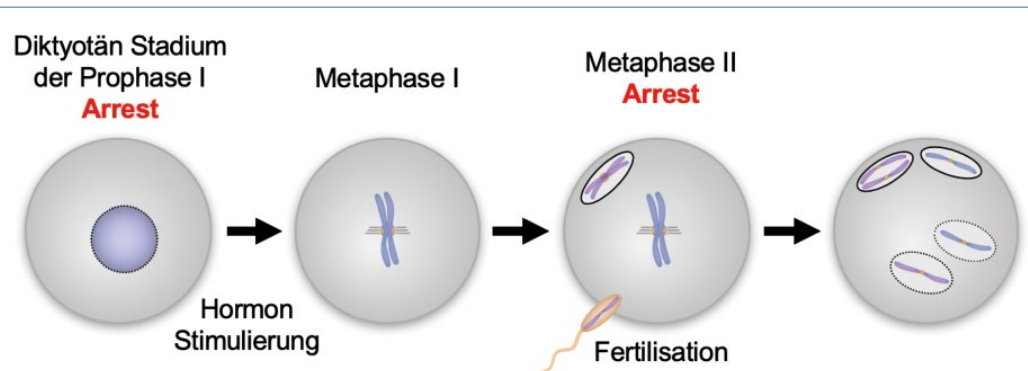

A Abb. 1: Oocyten durchlaufen zwei meiotische Teilungen. Unreife Oocyten sind im DiktyotänStadium der Prophase der ersten meiotischen Teilung (Prophase I) arretiert. Hormonstimulierung hebt diesen Zellzyklusarrest auf, und Oocyten treten wieder in die meiotische Teilung ein. In der Metaphase der ersten meiotischen Teilung (Metaphase I) werden homologe Chromosomen am Spindeläquator angeordnet und in der darauffolgenden Anaphase getrennt. Nach Eintritt in die zweite meiotische Teilung arretieren Oocyten in der Metaphase (Metaphase II). Dieser Arrest wird bei der Befruchtung aufgehoben.

Anzahl der entstehenden Oogonien von Spezies zu Spezies unterscheidet, haben alle Säugetiere gemeinsam, dass Eizellen nur einmalig während der Embryonalentwicklung gebildet und danach nicht erneuert werden. Dieser Umstand steht auch im kausalen Zusammenhang mit der Tatsache, dass das Risiko für die Geburt eines Embryos mit einer Trisomie, z. B. Trisomie 21, mit zunehmendem Alter der werdenden Mutter ansteigt [2].

\section{Oocyten arretieren in der Meiose}

Im Anschluss an die mitotische Teilungsphase entwickeln sich Oogonien zu unreifen Oocyten, die auf ihrem Weg zu einer befruchtungsfähigen Oocyte zwei meiotische Teilungen durchlaufen müssen. Während der ersten meiotischen Teilung kommt es dabei zur Trennung homologer Chromosomen, während in der zweiten meiotischen Teilung die Schwesterchromatiden getrennt werden. Nach Abschluss der Rekombination arretieren unreife Oocyten im Diktyotän-Stadium der Prophase der ersten meiotischen Teilung (Prophase I) mit gepaarten homologen Chromosomen (Abb. 1). Im Menschen wird dieses Ruhestadium bereits kurz nach der Geburt erreicht und erst bei Eintritt in die Pubertät wieder aufgehoben. Die Stimulation durch das luteinisierende Hormon führt dazu, dass eine Population unreifer Oocyten wieder in die Meiose eintritt und die erste meiotische Teilung beendet. Direkt im Anschluss treten Oocyten in die zweite meiotische Reifeteilung ein, wobei es in der Metaphase der Meiose II (MII) erneut zu einem Zellzyklusarrest kommt (Abb. 1). In diesem Stadium verharren die Oocyten - als reife Oocyten bis zur Befruchtung [2].

Mechanismus des Arrests reifer Oocyten in der Meiose II

Der Arrest reifer Oocyten in der Metaphase II zeichnet sich durch eine hohe Aktivität der Cyclin-abhängigen Kinase 1 (Cdk1) aus Cdk1 ist der master regulator der Zellzyklusregulation, dessen Aktivierung bzw. Inaktivierung für den Eintritt in bzw. den Austritt 


\section{Metaphase II arretierte Oozyte}
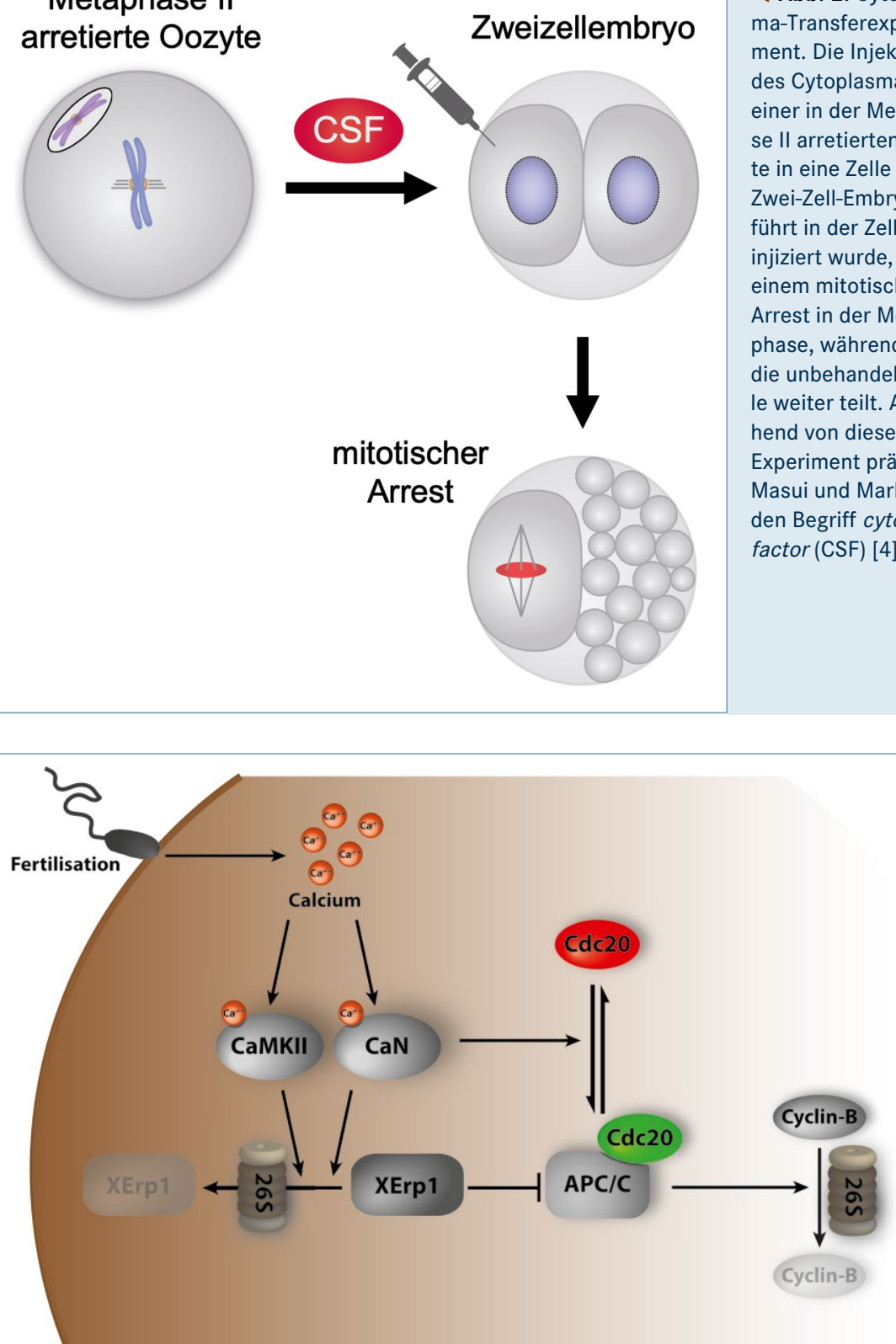

A Abb. 3: Mechanismus der Aktivierung des APC/C (anaphase promoting complex/cyclosome) bei der Befruchtung. Durch den transienten Calciumanstieg, ausgelöst durch die Befruchtung, werden die beiden calciumabhängigen Enzyme Calcium/Calmodulin-abhängige Kinase II (CaMKII) und Calcineurin (CaN) aktiviert. Dies führt dazu, dass CaMKII den APC/C-Inhibitor XErp1 dem proteasomalen Abbau zuführt. Somit kommt es zum APC/C-abhängigen Abbau von Cyclin B und Securin und letztendlich zum Vollenden der zweiten meiotischen Teilung. Aktiviertes CaN trägt zur Aktivierung des APC/C auf zwei Weisen bei: Zum einen unterstützt es den CaMKII-vermittelten Abbau von XErp1, und andererseits aktiviert es den APC/C-Ko-Aktivator Cdc20.

aus der meiotischen und mitotischen M-Phase verantwortlich ist. Die Aktivität von Cdk1 ist sowohl durch posttranslationale Modifikationen als auch durch die Assoziation mit aktivierenden Cyclin-Untereinheiten reguliert. Für die hohe Aktivität von Cdk1 in reifen Oocyten ist die Assoziation von B-TypCyclinen mit Cdk1 verantwortlich. Bei der Befruchtung kommt es zur Aktivierung der Ubiquitinligase APC/C (anaphase promoting complex/cyclosome), welche B-Typ-Cycline dem proteasomalen Abbau zuführt, was letztendlich die Inaktivierung von Cdk1 und somit den Austritt aus der M-Phase zur Folge hat [3].

Für den Arrest reifer Oocyten in der Metaphase II ist es demnach essenziell, dass der APC/C-vermittelte Abbau von B-Typ-Cyclinen verhindert wird. Erste Hinweise auf den zugrunde liegenden Mechanismus ergaben sich durch die bahnbrechenden Transferexperimente von Y. Masui und C. L. Markert in den 1970er-Jahren (Abb. 2, [4]). Dabei wurde Cytoplasma einer in der Metaphase II arretierten Oocyte des Leopardfroschs Rana pipiens in eine Zelle eines Zwei-Zell-Embryos injiziert. Während die unbehandelte Zelle ihre mitotischen Teilungen fortsetzte, arretierte die Zelle, die injiziert wurde, in der Metaphase der mitotischen Teilung. Aufgrund dieser Aktivität prägten Masui und Markert den Begriff cytostatic factor (CSF) [4]. Die molekulare Identität des CSF blieb jedoch trotz jahrzehntelanger Forschung unbekannt. Um diese Frage zu klären, nutzten wir den Krallenfrosch Xenopus laevis, einen gut etablierten Modellorganismus, für die Zellzyklusforschung. Unsere Arbeiten mit $X$. laevis identifizierten das Protein XErp1 als den gesuchten CSF. In vitro konnten wir zeigen, dass XErp1 die Ubiquitinligase-Aktivität des APC/C direkt inhibiert [5]. In Übereinstimmung mit seiner Funktion als CSF können Oocyten in Abwesenheit von XErp1 nicht in der Metaphase von MII arretieren, und die Injektion von XErp1 in embryonale Zellen induziert einen mitotischen MetaphaseArrest. Die Funktion von XErp1 als CSF ist evolutiv konserviert, da Mausoocyten in Abwesenheit von Emi2, dem Mausorthologen von XErp1, den Metaphase-II-Arrest nicht aufrechterhalten können [6].

\section{Inaktivierung von XErp1/Emi2 bei der Befruchtung}

Mit der Identifizierung von XErp1/Emi2 als CSF stellt sich die Frage, wie die Befruchtung den Metaphase-II-Arrest aufhebt. Bei der Befruchtung von Xenopus-Oocyten - wie auch bei Säugetieroocyten - kommt es zu einem plötzlichen Anstieg der intrazellulären Calciumkonzentration [7]. Dieser temporäre Anstieg der Calciumkonzentration aktiviert die Proteinkinase Calcium/Calmodulinabhängige Kinase II (CaMKII) [8]. Aktivierte CaMKII phosphoryliert XErp1 an einem Motiv, das als phosphorylierungsabhängiges Rekrutierungsmotiv für die polo-like kinase Plx1 dient. CaMKII funktioniert demnach als Priming-Kinase, welche die Rekrutierung von Plx1 an XErp1 induziert. An XErp1 gebundene Plx1 phosphoryliert ein Motiv, das als Erkennungsmotiv für eine weitere Ubiquitinligase, $\mathrm{SCF}^{\beta-T R C P}$, dient [9]. Diese führt XErp1 letztendlich dem proteasomalen Abbau zu, was zur Aktivierung des APC/C, dem Abbau der B-Typ-Cycline und somit zum Vollenden der zweiten meiotischen Teilung in den Oocyten führt.

\section{Weitere calciumabhängige Prozesse bei der Befruchtung}

In Xenopus-Oocyten führt der transiente Anstieg der intrazellulären Calciumkonzen- 
tration nicht nur zur Aktivierung der Kinase CaMKII, sondern auch der Proteinphosphatase Calcineurin (CaN). Durch die Kombination von in vitro-Untersuchungen mit Studien in Xenopus-Oocyten konnten wir zeigen, dass CaN die Aktivierung des APC/C durch zwei unabhängige, aber funktionell verzahnte Prozesse unterstützt: (1) CaN unterstützt den effizienten Abbau des APC/C-Inhibitors XErp1, und (2) fördert CaN die Aktivierung von Cdc20, einem KoAktivator des APC/C [10]. Durch diesen dualen Mechanismus unterstützt Calcineurin die Kinase CaMKII in ihrer Funktion, den APC/C bei der Befruchtung schnell und effizient zu aktivieren (Abb. 3).

\section{Ausblick}

Dank intensiver Forschungstätigkeiten in den vergangenen Jahren haben wir heutzutage ein sehr gutes molekulares Verständnis darüber, wie reife Oocyten in der Metaphase der zweiten meiotischen Teilung arretieren und wie dieser Arrest durch die Befruchtung aufgehoben wird. Die molekularen Mechanismen, die dem Arrest unreifer Oocyten in der Prophase der ersten meiotischen Teilung bzw. dem Hormon-induzierten Wiedereintritt in die Meiose zugrunde liegen, sind dagegen weitgehend ein Rätsel, das es zu lösen gilt. Um Einblicke in diese Mechanismen zu erlangen, haben wir bereits damit begonnen, neue, bislang unbekannte Substrate von Meioserelevanten Kinasen zu identifizieren. Durch die Kombination von Studien in XenopusOocyten mit in vitro-Analysen erwarten wir neuartige Einblicke in meiotische Regulationsmechanismen. Aufgrund der Tatsache, dass die grundlegenden Prozesse der meiotischen Teilung und deren Regulierung konserviert sind, gehen wir davon aus, dass sich die zu erwartenden Erkenntnisse auch auf Säugetiere übertragen lassen. Dieser Erkenntnisgewinn wäre somit nicht nur für die Grundlagenforschung von großem Interesse, sondern hat das Potenzial, auch für die Reproduktionsbiologie wichtige Einblicke zu liefern.

\section{Literatur}

[1] Sun YC, Sun XF, Dyce PW et al. (2017) The role of germ cell loss during primordial follicle assembly: a review of current advances. Int J Biol Sci 13:449-457

[2] Webster A, Schuh M (2017) Mechanisms of aneuploidy in human eggs. Trends Cell Biol 27:55-68 [3] Hormanseder E, Tischer T, Mayer TU (2013) Modulation of cell cycle control during oocyte-to-embryo transitions. EMBO J 32:2191-2203
[4] Masui Y, Markert CL (1971) Cytoplasmic control of nuclear behavior during meiotic maturation of frog oocytes. Journal Exp Zool 177:129-145

[5] Schmidt A, Duncan PI, Rauh NR et al. (2005) Xenopus polo-like kinase Plx1 regulates XErp1, a novel inhibitor of APC/C activity. Genes Dev 19:502-513

[6] Suzuki T, Suzuki E, Yoshida N et al. (2010) Mouse Emi2 as a distinctive regulatory hub in second meiotic metaphase. Development 137:3281-3291

[7] Busa WB, Nuccitelli R (1985) An elevated free cytosolic $\mathrm{Ca}^{2+}$ wave follows fertilization in eggs of the frog, Xenopus laevis. J Cell Biol 100:1325-1329

[8] Lorca T, Cruzalegui FH, Fesquet D et al. (1993) Calmodulin-dependent protein kinase II mediates inactivation of MPF and CSF upon fertilization of Xenopus eggs. Nature 366:270-273

[9] Rauh NR, Schmidt A, Bormann J et al. (2005) Calcium triggers exit from meiosis II by targeting the APC/C inhibitor XErp1 for degradation. Nature 437:1048-1052

[10] Heim A, Tischer T, Mayer TU (2018) Calcineurin promotes APC/C activation at meiotic exit by acting on both XErp1 and Cdc20. EMBO Rep 19, doi: 10.15252/embr. 201846433

\section{Funding: Open Access funding provided by Projekt DEAL.}

Open Access: Dieser Artikel wird unter der Creative Commons Namensnennung 4.0 International Lizenz veröffentlicht, welche die Nutzung Vervieffaltigung, Bearbeitung, Verbreitung und Wiedergabe in jeglichem die Quelle ordnungsgemäß nennen, einen Link zur Crüntive Commons Autor(en) und beifügen und angeben, ob Änderungen vorgenommen wurden. Die in diesem Artikel enthaltenen Bilder und sonstiges Drittmaterial unterliegen ebenfalls der genannten Creative Commons Lizenz, sofern sich aus der Abbildungslegende nichts anderes ergibt. Sofern das betreffende Material nicht unter der genannten Creative Commons Lizenz steht und die betreffende Handlung nicht nach gesetzlichen Vorschriften erlaubt ist, ist für die oben aufgeführten Weiterverwendungen des Materials die Einwilligung des jeweiligen Rechteinhabers einzuholen. Weitere Details zur Lizenz
entnehmen Sie bitte der Lizenzinformation auf http://creativecommons.org/

Korrespondenzadresse:

Prof. Dr. Thomas U. Mayer

Lehrstuhl für Molekulare Genetik

Universität Konstanz

Universitätsstraße 10

D-78467 Konstanz

thomas.u.mayer@uni.kn

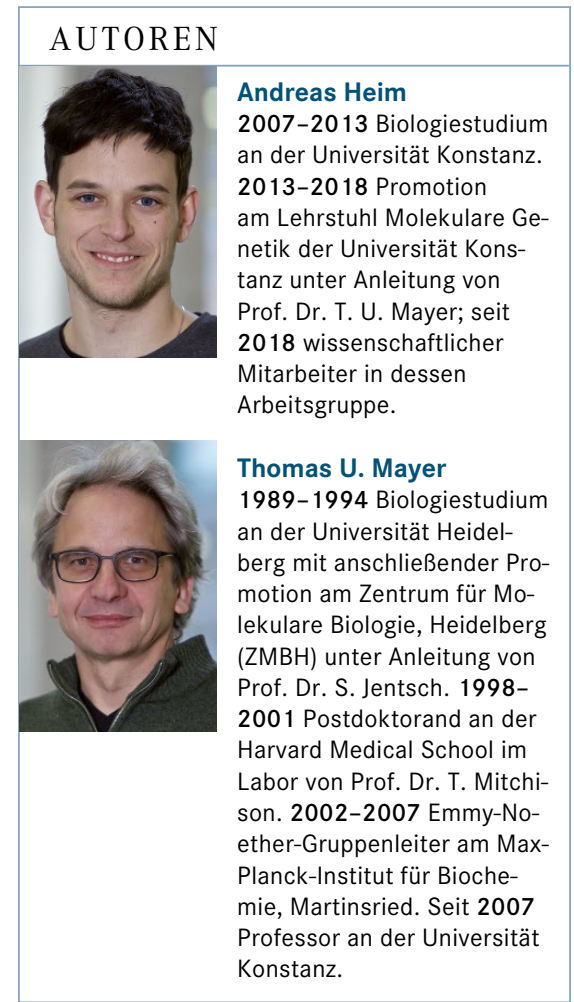

